\title{
Synthesis of a Novel Family of Polymeric Surfactants with Low Interfacial Tension by Ultrasonic Method
}

\author{
Ya CAO and Huilin LI \\ The State Key Laboratory of Polymer Materials Engineering, Polymer Research Institute of \\ Chengdu University of Science \& Technology, \\ Chengdu 610065, People's Republic of China
}

(Received March 11, 1999)

\begin{abstract}
A novel series of polymeric surfactants based on carboxy methyl cellulose and alkyl poly(etheroxy) acrylate was synthesized by ultrasonic irradiation. The structures of the copolymers were identified by viscometry, GPC, IR, DSC, and polarized light microscopy. The unique ultrasonic copolymerization mechanism renders a blocky nature to the copolymers prepared. The polymeric surfactants show excellent surface and interfacial properties.

KEY WORDS Polymeric Surfactant / Synthesis / Ultrasonic Irradiation /
\end{abstract}

Amphiphilic polymers consisting of hydrophilic and hydrophobic segments have found uses in many areas such as emulsifiers for stabilization of multiple emulsions, compatilizers in polymer blending, static electrical charge reducers and phase transfer catalysts, etc. These polymeric surfactants combine the desired properties of a polymer such as high viscosity with the interfacial property of a conventional surfactant. However, as high molecular weight would lead to weak ability to reduce the interfacial tension because of macromolecules entanglement, ${ }^{1}$ few polymeric surfactants that have both high molecular weight and low interfacial tension have been available.

In this study, a new approach was taken to synthesize a copolymer surfactant with low interfacial tension. Mechanochemical methods, such as ultrasonic irradiation, are convenient to obtain copolymers that combine different properties of the two polymers. ${ }^{2,3}$ When a polymer solution is subjected to ultrasonic irradiation, the polymer undergoes chain scission, thereby producing macroradicals. If another monomer is present in the solution, the monomer can be initiated by the macroradical and polymerized to yield a copolymer. Because of the unique polymerization mechanism, the copolymers thus produced are block copolymers. ${ }^{4}$

Some water-soluble polymers, such as polyacrylamide, modified cellulose are used extensively as thickening agents in areas such as enhanced oil recovery. These polymers usually possess high molecular weight and high viscosity. The water based polymers have advantages over oil based polymers in that they are environmentally friendly. Comb copolymers composed of long hydrophilic-hydrophobic chains have excellent interfacial activity. ${ }^{5}$ It is desired to synthesize a novel polymeric surfactant that combines the viscosity of the watersoluble polymers and the interfacial activity of the comb copolymers. This was achieved in the present study by adding a polymerizable surfactant with long hydrophilic-hydrophobic chains to aqueous carboxy methyl cellulose (CMC) solution which was then subjected to ultrasonic irradiation. Three different surface active macromonomers were prepared and copolymerized with CMC. To understand the ultrasonic reaction mechanism, ultrasonic irradiation of pure CMC was studied. The structures of the polymeric surfactants were examined by viscometry, ${ }^{13} \mathrm{C}$ NMR, GPC, IR, and DSC. The influence of structure on the surface /interfacial activity and viscosity of the copolymers was preliminarily investigated.

\section{EXPERIMENTAL}

\section{Materials}

Modified sodium CMC supplied by Guangzhou Nanxiu Chemical \& Engineering Co., was purified by acetone precipitation. The degree of $\mathrm{CH}_{2} \mathrm{COONa}$ substitution was 1.02 . The molecular weight was determined to be $\bar{M}_{n}=2.18 \times 10^{5}$ by viscosity measurements in $0.2 \mathrm{M} \mathrm{NaCl}$ solution using an Ubbelohde viscometer.

Surface active macromonomers, dodecyl poly(etheroxy) acrylate $\left(\mathrm{AR}_{12} \mathrm{EO}_{n}, n=3,9,20, n\right.$ the number of etheroxy units), were synthesized by reaction of acryloyl chloride with dodecyl poly(etheroxy) ether according to the preparation method reported in the literature. ${ }^{6}$

$$
\begin{gathered}
\mathrm{CH}_{2}=\mathrm{CHCOCl}+\mathrm{R}-\left(\mathrm{OCH}_{2} \mathrm{CH}_{2}\right)_{n}-\mathrm{OH} \rightarrow \\
\mathrm{CH}_{2}=\mathrm{CHCOO}-\left(\mathrm{CH}_{2} \mathrm{CH}_{2} \mathrm{O}\right)_{n}-\mathrm{R}+\mathrm{HCl} \\
\mathrm{R}=\mathrm{C}_{12} \mathrm{H}_{25}, \quad n=3,9,20
\end{gathered}
$$

\section{Apparatus}

Ultrasound with a frequency of $20 \mathrm{kHz}$ was produced with a Fisher Scientific 550 ultrasonic generator, the power output was $110 \mathrm{~W}$. The ultrasonic reactor is represented as Figure 1.

\section{Degradation Reaction of $C M C$}

Ultrasonic degradation of $\mathrm{CMC}$ was carried out in $0.6 \% \mathrm{CMC}$ aqueous solution in the presence of free radical terminator $\left(1.0 \times 10^{-3} \mathrm{~mol} \mathrm{~L}^{-1}\right.$ hydroquinone $)$ for different time periods. The reaction temperature was $25 \pm 1{ }^{\circ} \mathrm{C}$.

\section{Preparation and Purification of Polymeric Surfactants}

The polymeric surfactants were prepared by subjecting the aqueous solution of $0.6 \% \mathrm{CMC}$ in the presence of $0.5 \%$ surface-active macromonomers $\mathrm{AR}_{12} \mathrm{EO}_{n}$ to ultra- 


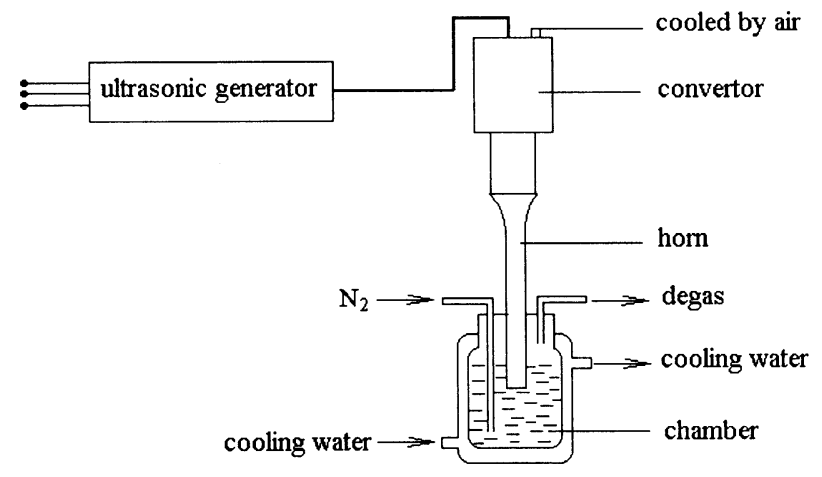

Figure 1. Ultrasonic reactor.

sonic irradiation for $20 \mathrm{~min}$ at $25 \pm 1^{\circ} \mathrm{C}$. The reaction mixture was dried and extracted by Soxhlet Apparatus with acetone for $36 \mathrm{~h}$ to get rid of unreacted macromonomers. The solid was dissolved in a mixture of $\mathrm{CH}_{3} \mathrm{OH}-\mathrm{H}_{2} \mathrm{O}(70: 30, \mathrm{v} / \mathrm{v})$. By adding $\mathrm{CH}_{3} \mathrm{CH}_{2} \mathrm{OH}$ to the solution, pure $\mathrm{CMC}$ was precipitated out while the copolymer was left in the solution. The solvent was evaporated and solid copolymer products were obtained. Finally, the products were purified by washing with acetone several times and drying under vacuum at $40^{\circ} \mathrm{C}$.

\section{Measurement}

$\bar{M}_{n}$ and intrinsic viscosity $[\eta]$ were determined by viscosity measurement in $0.2 \mathrm{M} \mathrm{NaCl}$ solution using an Ubbelohde viscometer at $25 \pm 0.1^{\circ} \mathrm{C}$. Aqueous solution viscosity was determined with a Brookfield TC500 viscometer at $30 \mathrm{rpm}$ using appropriate spindles.

The structure of the products was identified by ${ }^{13} \mathrm{C}$ NMR (Bruker AC-E200)

Molecular weight and molecular weight distribution were determined by Gel Permeation Chromatography (Waters 150C). The eluent, $0.2 \mathrm{M} \mathrm{NaNO}_{3}$ and $0.01 \mathrm{M}$ $\mathrm{NaH}_{2} \mathrm{PO}_{4}(\mathrm{pH}=7)$, was pumped through the column at a rate of $1.0 \mathrm{~mL} \mathrm{~min}^{-1}$. Volume of injection was $200 \mu \mathrm{L}$.

The infrared spectra were obtained on a Nicolet-560 Fourier Infrared Spectrophotomer. Each spectrum was recorded in double precision at a resolution $1 \mathrm{~cm}^{-1}$ with a total of 32 scans.

Differential scanning calorimeter spectra were obtained on Perkin-Elmer DSC-2C at a heating rate of $10^{\circ} \mathrm{C} \mathrm{m^{-1 }}$ from ambient temperature to $350^{\circ} \mathrm{C}$.

The crystal conformation of copolymers was observed by LEITZ LABORLUX 12POLS hot stage polarized light microscope, at a heating rate of $5^{\circ} \mathrm{C} \mathrm{min}{ }^{-1}$ from ambient temperature to $350^{\circ} \mathrm{C}$. The films of copolymers were made by vacuum drying of concentrated solutions.

Surface tension was measured by the plate method with Kruss-Kiost tensiometer at $25^{\circ} \mathrm{C}$. The accuracy is $\pm 0.1 \mathrm{mN} \mathrm{m}^{-1}$.

Interfacial tension measurements were carried out at $45^{\circ} \mathrm{C}$ on systems pre-equilibrated using Kruss SITE04 spinning drop tensiometer. Systems are aqueous solution of polymeric surfactants with Daqing crude oil $\left(\rho=0.87 \mathrm{~kg} \mathrm{~m}^{-3}\right)$. Interfacial tension $(I F T)$ was obtained by the equation:

$$
I F T=2.18899 \times 10^{-9} d_{0}{ }^{3} n^{2} f^{-1}\left(x_{0} / y_{0}\right)
$$

in which $d_{0}$ is diameter of an oil drop, $n$ rotating speed,

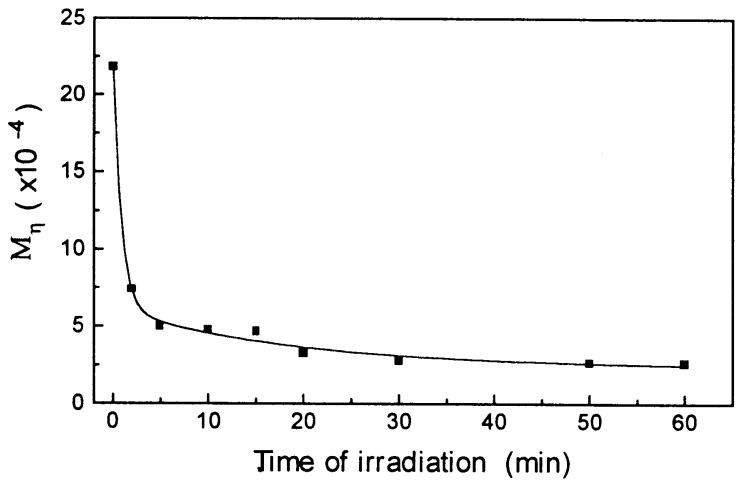

Figure 2. Molecular weight of CMC versus time of ultrasonic irradiation.



Figure 3. Carboxy methyl cellulose.

and $f^{-1}\left(x_{0} / y_{0}\right)$ corrector factor of shape. ${ }^{7}$

\section{RESULTS AND DISCUSSION}

\section{Ultrasonic Reactions}

Figure 2 shows the degradation curve of CMC. With increase of irradiation time, the molecular weight of CMC decreases. The decrease in molecular weight is rapid in the early stage of irradiation, but after $20 \mathrm{~min}$, the curve gradually levels off to a limiting value. This shows that degradation is fast for high molecular weight $\mathrm{CMC}$, but as the chains become shorter, the possibility of chain scission decreases.

According to the reported ESR results, ${ }^{8-11}$ three macroradicals are generated during the mechanical degradation of cellulose. These are radicals derived from $\mathrm{C}_{2}, \mathrm{C}_{3}, \mathrm{C}_{1}$, and $\mathrm{C}_{4}$ positions of the cellulose (Figure 3 ). Of these radicals, alkoxy radicals derived from $\mathrm{C}_{1}$ and $\mathrm{C}_{4}$ positions are the most stable at ambient temperature. This indicates that the main fashion of CMC degradation is the cleavage of CMC backbones, which leads to deep decrease in the molecular weight. Therefore the macroradicals at $\mathrm{C}_{1}$ and $\mathrm{C}_{4}$ positions of $\mathrm{CMC}$ are the main radicals that initiate active monomers.

\section{Identification Block Copolymers}

The ${ }^{13} \mathrm{C}$ NMR spectrum of the purified product $\mathrm{CMC}-\mathrm{AR}_{12} \mathrm{EO}_{9}$ is shown in Figure 4. Compared with the ${ }^{13} \mathrm{C}$ NMR spectrum of $\mathrm{CMC},{ }^{12}$ new peaks[chemical shift: $9.3\left(\mathrm{CH}_{3}\right), 28.2\left(\mathrm{CH}_{2}\right), 30.9\left(\mathrm{CH}_{2}\right), 73.0-72.4$ $\left.\left(\mathrm{CH}_{2} \mathrm{CH}_{2} \mathrm{O}\right)\right]$ attributed to the macromonomer segments ${ }^{13}$ show up in addition to the CMC peaks. Because the unreacted macromonomers had been washed off by acetone, the macromonomer segments integral parts in the products, thus proving that ultrasonic copolymerization occurs.

The structural characteristics of the copolymers are listed in Table I. The composition of the copolymers was determined by titration of the COONa groups of CMC 


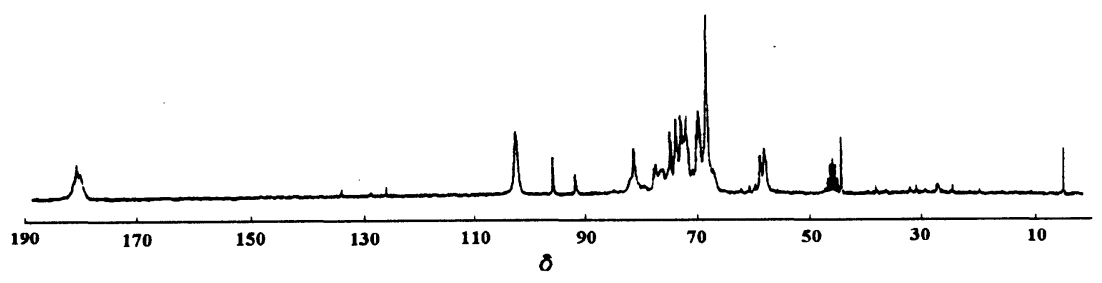

Figure 4. ${ }^{13} \mathrm{C}$ NMR spectrum of $\mathrm{CMC}-\mathrm{AR}_{12} \mathrm{EO}_{9}$.

Table I. Structural characteristics of polymeric surfactants

\begin{tabular}{|c|c|c|c|c|}
\hline \multirow{2}{*}{ Sample } & \multirow{2}{*}{ System } & \multirow{2}{*}{$\begin{array}{l}\text { Macromonomer } \\
\text { composition/wt } \%\end{array}$} & \multirow{2}{*}{$\begin{array}{l}\bar{M}_{w} \\
\times 10^{-4}\end{array}$} & {$[\eta]$} \\
\hline & & & & $\mathrm{mLg}^{-1}$ \\
\hline B3 & $\mathrm{CMC}-\mathrm{AR}_{12} \mathrm{EO}_{3}$ & 6 & 8.8 & 470 \\
\hline B9 & $\mathrm{CMC}-\mathrm{AR}_{12} \mathrm{EO}_{9}$ & 10 & 16.9 & 381 \\
\hline B20 & $\mathrm{CMC}-\mathrm{AR}_{12} \mathrm{EO}_{20}$ & 7 & 19.6 & 304 \\
\hline
\end{tabular}

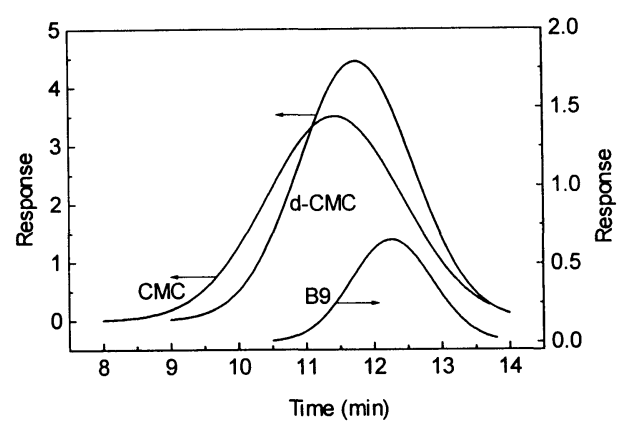

Figure 5. Elution curves in GPC of $0.1 \% \mathrm{CMC}, \mathrm{CMC}$ degraded (d-CMC), and $0.02 \% \mathrm{~B} 9$ at $25^{\circ} \mathrm{C}$.

with acid, and the molecular weight $\left(\bar{M}_{w}\right)$ was determined by static laser scattering.

The GPC patterns of $0.1 \%$ CMC, $d$-CMC (CMC aqueous solution irradiated for $20 \mathrm{~min}$ in the absence of a terminator) and $0.02 \%$ copolymer (B9) are shown in Figure 5. (To avoid aggregation of polymeric surfactants in salt solution, concentration of the B9 copolymer used in GPC was lower than its critical micelle concentration.) The molecular weight of $\mathrm{CMC}$ decreases after irradiation. Also the molecular weight distribution becomes narrower, which indicates that the cleavage of long chains occurs more frequently than short chains. Comparing the elution curve of $d$-CMC with that of $\mathrm{B} 9$, both irradiated at the same conditions, B9 has a lower molecular weight and a narrower molecular weight distribution. This shows that the scission of long CMC chains occurs and reactions between CMC macroradicals and the macromonomers are favored over recombination reactions between CMC macroradicals.

The Fourier infrared spectra of $\mathrm{CMC}$ and $\mathrm{B} 9$ are shown in Figure 6. Also shown in this figure is the spectrum of the grafting copolymer, $\mathrm{Ce}-\mathrm{CMC}-\mathrm{AR}_{12} \mathrm{EO}_{9}$, prepared chemically by reacting $\mathrm{CMC}$ with macromonomers with ceric compound $\left(\mathrm{Ce}^{4+}\right)$ as the initiator. ${ }^{14,15}$ In the chemical method, CMC macroradicals are generated by the interaction of $\mathrm{Ce}^{4+}$ with $-\mathrm{OH}$ groups at the $\mathrm{C}_{2}$ and $\mathrm{C}_{3}$ positions of $\mathrm{CMC}$ molecules and macroradicals initiate active monomers to yield grafting copolymers. The reaction mechanism is different from that of the ultrasonic reactions.

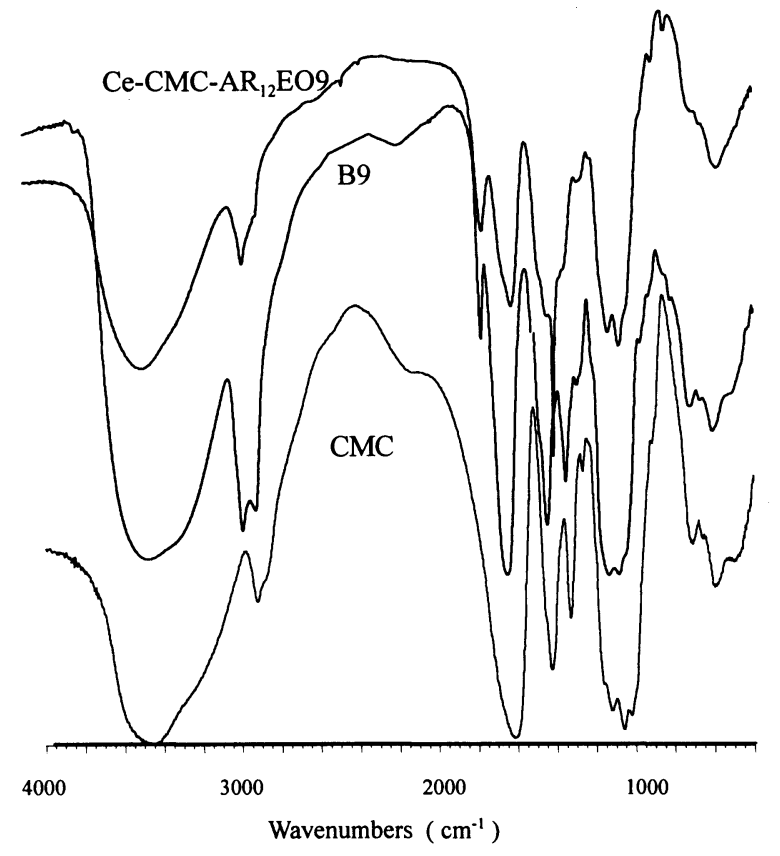

Figure 6. FT-IR spectra of CMC, $\mathrm{Ce}-\mathrm{CMC}-\mathrm{AR}_{12} \mathrm{EO}_{9}$, and $\mathrm{B} 9$.

Table II. Transition temperatures and enthalpies of $\mathrm{CMC}, \mathrm{Ce}-\mathrm{CMC}-\mathrm{AR}_{12} \mathrm{EO}_{9}$, and $\mathrm{B} 9$ in DSC

\begin{tabular}{cccc}
\hline & $\mathrm{CMC}$ & $\mathrm{B} 9$ & $\mathrm{Ce}-\mathrm{CMC}-\mathrm{AR}_{12} \mathrm{EO}_{9}$ \\
\hline $\mathrm{T} 1 /{ }^{\circ} \mathrm{C}$ & 287.2 & 258.2 & 195.4 \\
$\Delta H / \mathrm{Jg}^{-1}$ & -99.8 & -95.0 & -140.9 \\
\hline
\end{tabular}

The spectra of the copolymers $\mathrm{B} 9$ and $\mathrm{Ce}-\mathrm{CMC}-$ $\mathrm{AR}_{12} \mathrm{EO}_{9}$ are almost same as that of CMC because of the majority of $\mathrm{CMC}$ segments in the copolymers. However, the spectrum of $\mathrm{Ce}-\mathrm{CMC}-\mathrm{AR}_{12} \mathrm{EO}_{9}$ shows variation in the range $1300-1450 \mathrm{~cm}^{-1}$ where the bands belong to $\mathrm{C}-\mathrm{O}$ vibrations. The changes can be attributed to the grafting reactions that occur at $\mathrm{C}_{2}-\mathrm{OH}$ and $\mathrm{C}_{3}-\mathrm{OH}$ of cellulose. No such changes are observed in the $\mathrm{B} 9$ spectrum, thus essentially excluding the possibility of grafting reaction at the $\mathrm{C}_{2}$ or $\mathrm{C}_{3}$ positions in the ultrasonic copolymerization. The existence of macromonomer segments in the $\mathrm{B} 9$ copolymer dose not change the CMC spectrum significantly. This is in agreement with the fact that ultrasonic copolymerization yields block copolymers.

DSC measurements show that $\mathrm{CMC}$, B9, and $\mathrm{Ce}-$ CMC- $\mathrm{AR}_{12} \mathrm{EO}_{9}$ all exhibit only one exothermal peak from ambient temperature to $350^{\circ} \mathrm{C}$, which may result from the orientation and crystallization of macromolecules. The transition temperatures and enthalpies are listed in Table II. In the hot stage polarized 




Figure 7. Polarization microphotograph of $\mathrm{B} 9(\times 1000$, cross $)$.

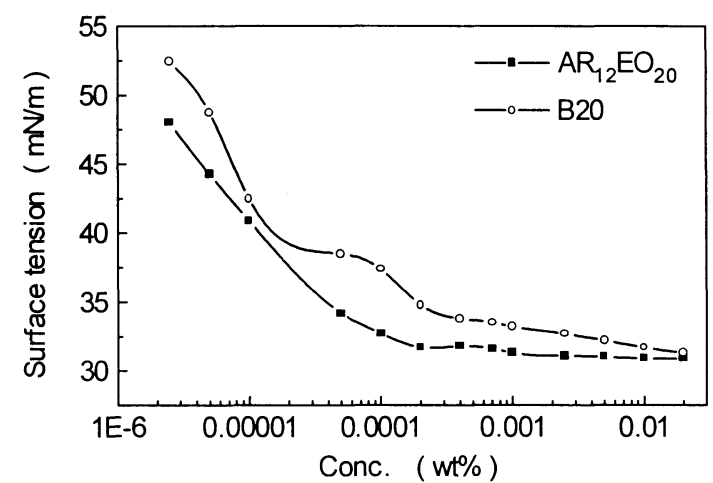

Figure 8. Surface tension isotherms of low molecular weight surfactant $\mathrm{AR}_{12} \mathrm{EO} 20$ and copolymer B20.



Figure 9. Influence of concentration on interfacial tension of macromonomer $\mathrm{AR}_{12} \mathrm{EO} 20$ and copolymer B20.

Table III. Surface / interfacial tension and viscosity of $0.5 \%$ copolymer aqueous solutions

\begin{tabular}{lrrr}
\hline & B3 & B9 & B20 \\
\hline Surface tension $/ \mathrm{mN} \mathrm{m}^{-1}$ & 33.7 & 30.4 & 32.5 \\
Interfacial tension $/ \mathrm{mN} \mathrm{m}^{-1}$ & 3.2 & 2.7 & 1.9 \\
$\eta_{\mathrm{a}} / \mathrm{mPa} \mathrm{s}$ & 12.9 & 10.4 & 12.1 \\
\hline
\end{tabular}

light microscopic measurements, the samples CMC, B9, and $\mathrm{Ce}-\mathrm{CMC}-\mathrm{AR}_{12} \mathrm{EO}_{9}$ show no melting state and carbonize at $310^{\circ} \mathrm{C}, 290^{\circ} \mathrm{C}$, and $250^{\circ} \mathrm{C}$, respectively. However, below the carbonizing temperature, sand-like or star-like structure appears under cross polarized light (Figure 7), presence of orientation or crystallization structure.

Thermal transitions can be attributed to order change in the CMC molecules when the intra- and intermolecular hydrogen bonds are destroyed at high temperature. The thermal orientation of $\mathrm{Ce}-\mathrm{CMC}-$ $\mathrm{AR}_{12} \mathrm{EO}_{9}$ occurs at a lower temperature because the hydrogen bonds are hindered by long grafting chains. The exothermal peak of $\mathrm{B} 9$ is close to that of $\mathrm{CMC}$, which indicates that the main structure of the ultrasonic copolymers is a block.

\section{Properties of the Polymeric Surfactants}

As a water-soluble polymer, the original $\mathrm{CMC}$ or $d$-CMC has weak surface activity.The surface tension of $0.5 \% \mathrm{CMC}$ aqueous solution is $64.5 \mathrm{~m} \mathrm{~N} \mathrm{~m}^{-1}$. The surface activity is greatly improved after reaction with surface active macromonomers. The surface tension and interfacial tension isotherms of the low molecular weight surfactant (macromonomer $\mathrm{AR}_{12} \mathrm{EO}_{20}$ ) and copolymer B20 are shown in Figures 8 and 9 respectively. Surface tension and IFT of the copolymers are slightly higher than those of the low molecular weight surfactants (L-surfactants). However, with increase of surfactant concentration, the difference in tension values between the copolymer surfactant and L-surfactant diminishes until finally the same value is reached.

The surface/interfacial tension and viscosity of $0.5 \%$ aqueous solutions of copolymers B3, B9, and B20 are listed in Table III. All the three copolymers have low surface and interfacial tension, nearly comparable to those of L-surfactants. The copolymer surfactants have high viscosity owing to the CMC blocks. The high molecular weight of the polymeric surfactants has only limited influence on surface and interfacial activity. The excellent properties displayed by the polymeric surfactants result from the special blocky structure obtained through ultrasonic irradiation. CMC blocks contribute to the high viscosity while surface active macromonomer blocks contribute to the high surface activity.

Acknowledgment. This project was funded by the State Scaling Height Foundation.

\section{REFERENCES}

1. M. R. Porter, "Handbook of Surfactants," Blackie Academic \& Professional, New York, N.Y., 1993, p 211.

2. C. Antonio, P. Rogers, "Polymer Stress Reaction," Academic Press, New York, N.Y., 1978, pp 8-18.

3. K. Q. Chen, Y. Shen, H. L. Li, and X. Xu, J. Macromol. Sci.-Chem., A22, 455 (1985).

4. H. Fujiware, K. Kimura, H. Mori, and K. Goto, Polym. J., 13, 927 (1981).

5. Y. Baraket, T. S. Gendy, A. I. Mohamad, and A.-F. M. Youssef, Br. Polym. J., 21, 383, (1989); Y. Baraket, I. K. Basily, A. I. Mohamad, and A. M. Youssef, ibid., 21, 459 (1989).

6. J. Diekstein, US Patent, 4075411 (1978).

7. H. M. Princen, I. Y. Z. Zia, and S. G. Mason, J. Colloid Interface Sci., 23, 99 (1967).

8. B. Randy and J. F. Rabek, "ESR Spectroscopy in Polym. Research," Springer-Verlag, Berlin, Heidelberg, 1977.

9. C. Jett, J. R. Arthur, and H. Oscar, J. Polym. Sci., Part C, 36, 53 (1971).

10. David and N. S. HON, J. Appl. Polym. Sci., 23, 1478 (1979).

11. David and N. S. HON, "ACS Sym. Ser.," American Chemical Society, Washington, D.C., 1983, pp 259-279.

12. S. Gautier and J. Lecourtier, Polym. Bull., 26, 457 (1991).

13. A. A. Rlbelro and E. A. Dennls, J. Phys. \& Chem., 81, 957 (1977).

14. N. W. Taylor and G. F. Fanta, J. Appl. Polym. Sci., 22, 1343 (1977).

15. N.Mohanty, B. Prodham, N. C. Mohanta, and H. K. Das, J. Macromol. Sci.-Chem., A19, 1189 (1983). 